\title{
Role of sensory and cognitive conspicuity in the prevention of collisions between motorcycles and trucks at $\mathrm{T}$-intersections
}

\begin{abstract}
Motorcyclists are particularly vulnerable to injury in crashes with heavy vehicles due to substantial differences in vehicle mass, the degree of protection and speed. There is a considerable difference in height between motorcycles and trucks; motorcycles are viewed by truck drivers from downward angles, and shorter distances between them mean steeper downward angles. Hence, we anticipated that the effects of motorcycle conspicuity treatments would be different for truck drivers. Therefore, this study aims to evaluate the effects of motorcycle conspicuity treatments on the identification and detection of motorcycles by truck drivers. Two complementary experiments were performed; the first experiment assessed the impact of motorcycle sensory conspicuity on the ability of un-alerted truck drivers to detect motorcycles, and the second experiment assessed the motorcycle cognitive conspicuity to alerted truck drivers. The sensory conspicuity was measured in terms of motorcycle detection rates by un-alerted truck drivers when they were not anticipating a motorcycle within a realistic driving scene, while the cognitive conspicuity was determined by the time taken by alerted truck drivers to actively search for a motorcycle. In the first experiment, the participants were presented with 10 pictures and were instructed to report the kinds of vehicles that were presented in the pictures. Each picture was shown to the participants for $600 \mathrm{~ms}$. In the second experiment, the participants were presented with the same set of pictures and were instructed to respond by clicking the right button on a mouse as soon as they detected a motorcycle in the picture. The results indicate that the motorcycle detection rate increases, and the response time to search for a motorcycle decreases, as the distance between the targeted motorcycle and the viewer decreases. This is true regardless of the type of conspicuity treatment used. The use of daytime running headlights (DRH) was found to increase the detection rate and the identification of a motorcycle by a truck driver at a farther distance, but effect deteriorates as the distance decreases. The results show that the detection rate and the identification of a motorcyclist wearing a black helmet with a reflective sticker increases as the distance between the motorcycle and the truck decreases. We also found that a motorcyclist wearing a white helmet and a white outfit is more identifiable and detectable at both shorter and longer distances. In conclusion, although this study provides evidence that the use of appropriate conspicuity treatments enhances motorcycle conspicuity to truck drivers, we suggest that more attention should be paid to the effect of background environment on motorcycle conspicuity.
\end{abstract}

Keyword: Motorcycle conspicuity; Sensory and cognitive conspicuity; Look-but-fail-to-see errors; Trucks 